\title{
Coherent Optical Creation of a Single Molecule
}

\author{
Yichao Yu $\odot,{ }^{1,2,3, \dagger}$ Kenneth Wang, ${ }^{1,2,3, \dagger}$ Jonathan D. Hood, ${ }^{4,4}$ Lewis R. B. Picard $\odot,{ }^{1,2,3}$ Jessie T. Zhang, ${ }^{1,2,3}$ \\ William B. Cairncross, ${ }^{2,1,3}$ Jeremy M. Hutson $\odot,{ }^{5}$ Rosario Gonzalez-Ferez, ${ }^{6,7}$ \\ Till Rosenband $\oplus^{8}$, and Kang-Kuen $\mathrm{Ni}^{2,1,3, *}$ \\ ${ }^{1}$ Department of Physics, Harvard University, Cambridge, Massachusetts 02138, USA \\ ${ }^{2}$ Department of Chemistry and Chemical Biology, Harvard University, \\ Cambridge, Massachusetts 02138, USA \\ ${ }^{3}$ Harvard-MIT Center for Ultracold Atoms, Cambridge, Massachusetts 02138, USA \\ ${ }^{4}$ Department of Chemistry, Purdue University, West Lafayette, Indiana 47906, USA \\ ${ }^{5}$ Joint Quantum Centre Durham-Newcastle, Department of Chemistry, Durham University, \\ Durham, DH1 3LE, United Kingdom \\ ${ }^{6}$ Instituto Carlos I de Física Teórica y Computacional, and Departamento de Física Atómica, \\ Molecular y Nuclear, Universidad de Granada, 18071 Granada, Spain \\ ${ }^{7}$ ITAMP, Harvard-Smithsonian Center for Astrophysics, Cambridge, Massachusetts 02138, USA \\ ${ }^{8}$ Agendile LLC, Cambridge, Massachusetts 02139, USA
}

(Received 17 December 2020; revised 11 May 2021; accepted 12 July 2021; published 17 September 2021)

\begin{abstract}
We report coherent association of atoms into a single weakly bound $\mathrm{NaCs}$ molecule in an optical tweezer through an optical Raman transition. The Raman technique uses a deeply bound electronic excited intermediate state to achieve a large transition dipole moment while reducing photon scattering. Starting from two atoms in their relative motional ground state, we achieve an optical transfer efficiency of $69 \%$. The molecules have a binding energy of 770.2 MHz at 8.83(2) G. This technique does not rely on Feshbach resonances or narrow excited-state lines and may allow a wide range of molecular species to be assembled atom by atom.
\end{abstract}

DOI: 10.1103/PhysRevX.11.031061

Diverse species of fully quantum-controlled ultracold molecules are desired for a variety of applications including precision measurements [1-6], quantum simulations [7-10], quantum information processing [11-14], and studies of ultracold chemistry [15-18]. While many innovative approaches in the past few years have directly cooled different species of molecules below $1 \mathrm{mK}$ [19-24], the highest phase-space-density gas [25] and trapped individual molecules $[26,27]$ have been achieved through the association of ultracold atoms.

Molecular association of ultracold atoms takes advantage of the cooling and trapping techniques that have been developed for atoms. Associating atoms into deeply bound molecules is challenging because of the small wave function overlap between the free-atom and molecular states and the release of large binding energy. A widely used method of overcoming these challenges is to associate

\footnotetext{
* To whom correspondence should be addressed. ni@chemistry.harvard.edu

These authors contributed equally to this work.

Published by the American Physical Society under the terms of the Creative Commons Attribution 4.0 International license. Further distribution of this work must maintain attribution to the author(s) and the published article's title, journal citation, and DOI.
}

Subject Areas: Atomic and Molecular Physics

atom pairs into weakly bound molecules first, and then transfer the molecules from this single internal state to a desired rovibrational and electronic state, releasing the binding energy by stimulated emission [1,28-35]. So far, coherent molecular association has generally been achieved by magnetoassociation through a magnetic Feshbach scattering resonance. Exceptions include $\mathrm{Sr}_{2}$, where narrow linewidth $(\sim 20 \mathrm{kHz})$ excited states are available and optical association can be driven coherently [36,37], and ${ }^{87} \mathrm{Rb}^{85} \mathrm{Rb}$ with molecular states bound by only $1-2 \mathrm{MHz}$ [27]. With these requirements, molecules involving nonmagnetic atoms [38] or atoms without narrow intercombination lines remain difficult to create. Optical association that can be applied to a wider range of molecules has also been demonstrated [39-41]. Although these attempts use a pair of laser beams to target a particular molecular state, the created molecule was lost quickly before any coherent Rabi oscillations were observed. This technique can be used for spectroscopic study of the molecular structure and interactions, but without control of the created molecule, further transfer to states of interest in quantum computing or simulation remains difficult.

Here, we demonstrate coherent association of an atom pair to a weakly bound molecule by two-photon optical Raman transfer via an electronic excited state, as shown in Fig. 1(a), taking $\mathrm{NaCs}$ as the prototype system. We observe 
coherent Rabi oscillations between the free atoms and the weakly bound molecule. The technique does not rely on a Feshbach resonance, molecular states bound by only a few megahertz, or a narrow excited state. The resulting single molecule is in a well-defined internal quantum state and predominantly in its motional ground state. A vibrational state of the electronic excited state $c^{3} \Sigma^{+}(\Omega=1)$ serves as the intermediate state in the Raman technique, and is chosen to minimize photon scattering during Raman Rabi oscillations. To reduce photon scattering and sensitivity to laser intensity noise further, we choose the initial and final states that balance the two Rabi frequencies as much as possible. This approach applies to a variety of molecules that can be created atom by atom with full quantum-state control.

The optical Raman transfer is illustrated by the idealized three-level system shown in Fig. 1(a), where the initial atomic state and the target weakly bound molecular state are coupled to an intermediate state by two lasers with Rabi frequencies $\Omega_{a}$ and $\Omega_{m}$, one-photon detuning $\Delta$, and all Rabi frequencies are population oscillation frequencies. The transfer Raman Rabi frequency is given by $\Omega_{a} \Omega_{m} /(2 \Delta)$ [42]. Unlike Raman transitions in atoms, the one-photon Rabi frequencies are greatly imbalanced $\left(\Omega_{a} / \Omega_{m} \ll 1\right)$ due to the small wave function overlap between the atomic state and the intermediate state, and scattering losses are dominated by the final state. Because the energy difference between the atomic state and target molecular state is small $(<1 \mathrm{GHz})$ compared to the singlephoton detuning of 80-200 GHz, both beams scatter nearly equally with a total rate $\Gamma_{e} \Omega_{m}^{2} /\left(2 \Delta^{2}\right)$, where $\Gamma_{e}$ is the excited-state linewidth. The two beams have equal power to maximize the Raman Rabi frequency at fixed total power. In this idealized treatment, the ratio between the Raman Rabi frequency and the scattering rate is $\Omega_{a} / \Omega_{m} \times \Delta / \Gamma_{e}$, which limits the transfer efficiency into the molecular state. At the same time, the intensity-stability requirement is determined by the ratio of Raman Rabi frequency to light shift $\Omega_{a} / \Omega_{m}$. Notably, both figures of merit improve with a larger ratio $\Omega_{a} / \Omega_{m}$.

Earlier experiments used excited states with high vibrational quantum number for the intermediate state of the Raman transition to ensure a large Raman Rabi frequency $[39,40]$. However, a complete picture includes both the many vibrational levels of the excited electronic state and the atomic continuum. Then the scattering and Raman Rabi rates are sums over all possible intermediate states. As there is large overlap between the target molecular state and other vibrationally excited states, intermediate states that are closer to the dissociation threshold result in a large scattering rate. This scattering is approximately proportional to $1 / \Delta_{\text {thresh }}^{2}$, where $\Delta_{\text {thresh }}$ is the detuning from the dissociation threshold, which is smaller for intermediate states that are deeply bound.

We optimize over intermediate states by calculating the total Raman Rabi frequency $\Omega_{R}$ and scattering rate $\Gamma_{s}$ at different detunings from the atomic threshold, taking into account all states of 8 excited molecular electronic potentials [43-47] and the continuum [48]; see the Appendix B for details. This calculation shows that the figure of merit $\Omega_{R} / \Gamma_{s}$ can be larger for the lowest vibrational state
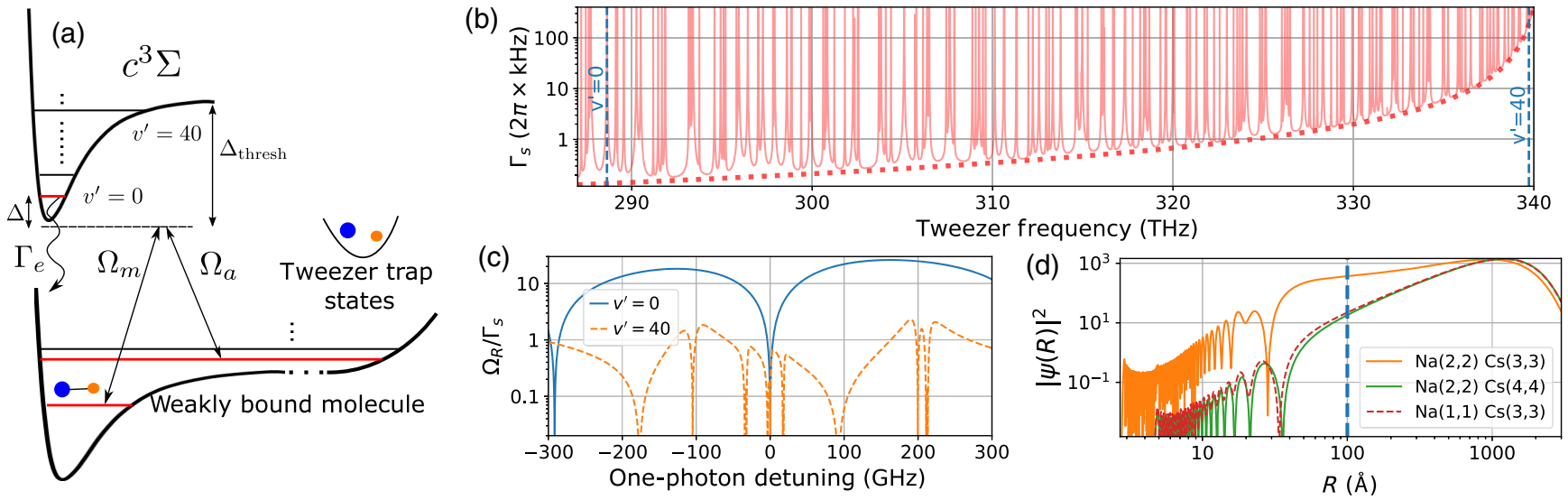

FIG. 1. Optical creation of single molecules from single atoms in an optical tweezer. (a) Schematic of the optical transition from an atom pair to a weakly bound molecule. The initial state is the relative motional ground state between the two atoms and the final state is the first molecular bound state. The transition is driven by a pair of laser frequencies whose difference $(<1 \mathrm{GHz})$ matches the molecular binding energy. The lasers are detuned by $\Delta$ from an excited vibrational sublevel in the $c^{3} \Sigma^{+}(\Omega=1)$ electronic state in order to reduce scattering during the transfer. (b) Calculation of the scattering rate versus laser frequency including all vibrational states of 8 excitedstate potentials and the atomic continuum. The assumed excited-state linewidth for molecular lines is $50 \mathrm{MHz}$. The dotted red line shows the contribution from the near-threshold states, which approximately scales as $1 / \Delta_{\text {thresh. }}^{2}$ (c) Comparison between vibrational sublevels of the intermediate excited state for the Raman transition. The lowest sublevel (blue line) has a larger optimum ratio of Raman Rabi frequency to scattering rate than the higher sublevel (orange line). (d) The large scattering length for $\mathrm{Na}(2,2), \mathrm{Cs}(3,3)$ is associated with enhancement of the relative wave function at short internuclear distance $(R)$. 
compared to higher bound states at a cost of a smaller transfer rate $\Omega_{R}$, as shown in Fig. 1(c). As a result, we choose the $v^{\prime}=0$ level of $c^{3} \Sigma^{+}(\Omega=1)$ as the intermediate state near which to drive Raman transitions.

In addition to the intermediate state, the choice of initial and final Zeeman and hyperfine states affects the singlephoton rates $\Omega_{a}$ and $\Omega_{m}$. Because of the small extent of the intermediate-state wave function compared to that of the trapped atoms, $\Omega_{a}$ is approximately proportional to the amplitude of the relative atomic wave function at short distance, within the range of the molecular potential. To increase this amplitude, one can increase the external confinement of atom pairs. In a harmonic approximation the short-range amplitude is proportional to $\omega_{\text {trap }}^{3 / 4}$ or $P^{3 / 8}$, where $\omega_{\text {trap }}$ is the trap frequency and $P$ is the optical power in the tweezer trap [49] with a fixed beam waist. However, additional power may not be available and also leads to additional undesired scattering. Alternatively, one can choose an atomic pair state with a large scattering length (positive or negative). For such states, the amplitude of the relative atomic wave function is substantially enhanced at short range, as shown in Fig. 1(d). For our system of $\mathrm{Na}$ and Cs atoms, we choose a spin-state combination $\left|\uparrow_{\mathrm{Na}} \downarrow_{\mathrm{Cs}}\right\rangle \equiv$ $\left|f=2, m_{f}=2\right\rangle_{\mathrm{Na}}\left|f=3, m_{f}=3\right\rangle_{\mathrm{Cs}}$ that has a large and negative scattering length of $a\left(\uparrow_{\mathrm{Na}} \downarrow_{\mathrm{Cs}}\right) \approx-700 a_{0}$ [50].

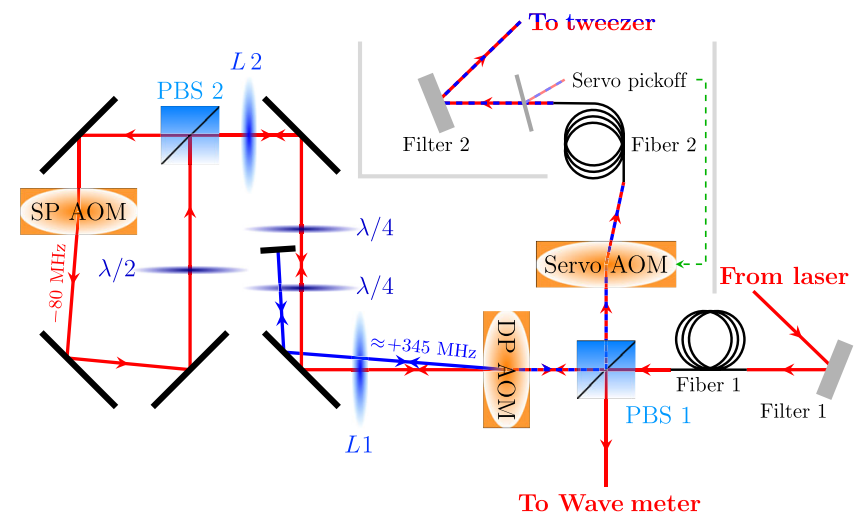

FIG. 2. Beam path for generating two frequencies in the tweezer for the Raman transition (only essential optics shown). Two filters, one directly after the laser source and one after both optical fibers, clean the light from a fiber amplifier seeded by an external cavity diode laser. The red beam path is the alwayspresent zeroth order of the double pass (DP) AOM. The blue beam path is the switchable first order of the DP AOM. It generates the second frequency for the Raman transition. To reduce interferences that cause relative power fluctuation of the two frequencies, the zeroth order is frequency shifted by $-80 \mathrm{MHz}$ before recombination. The experiment typically starts with the single-pass (SP) AOM on and the DP AOM off. When driving the Raman transition, the rf powers for both AOMs are ramped simultaneously to achieve the desired optical power at both frequencies, while keeping the total optical power fixed. Abbreviations: PBS-polarization beam splitter and AOMacousto-optic modulator.
All other stable spin combinations give smaller scattering lengths $\left(<50 a_{0}\right)$.

To identify a suitable target molecular state, we carry out coupled-channel calculations of the near-threshold bound states, as described in Appendix E. Choosing a bound state with similar spin character to the atomic state minimizes the sensitivity of the transition frequency to magnetic field. A suitable state with this character is predicted about $763 \mathrm{MHz}$ below the $\left|\uparrow_{\mathrm{Na}} \downarrow_{\mathrm{Cs}}\right\rangle$ threshold and the ratio $\Omega_{a} / \Omega_{m}$ increases to about 0.013 . Compared to $\Omega_{a} / \Omega_{m} \approx$ 0.003 for other combinations, this relaxes the intensity

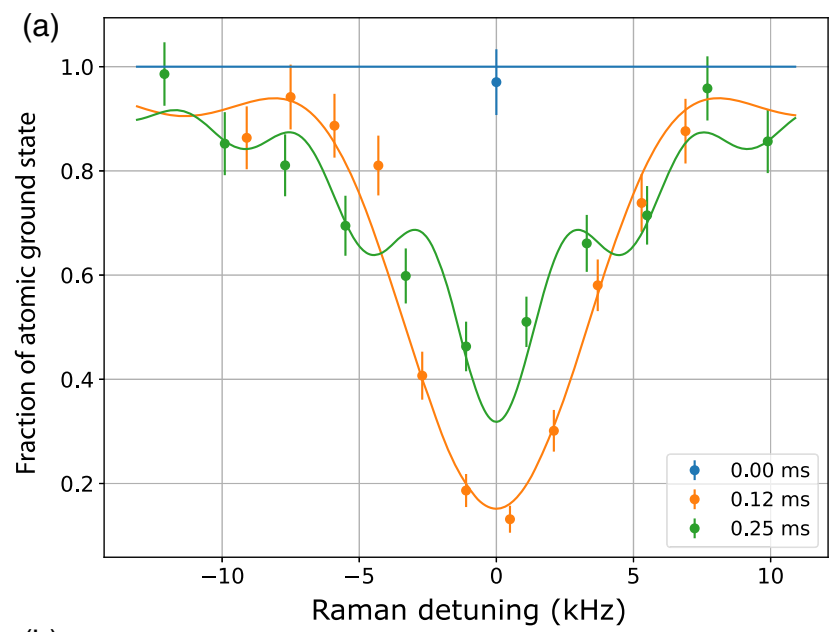

(b)

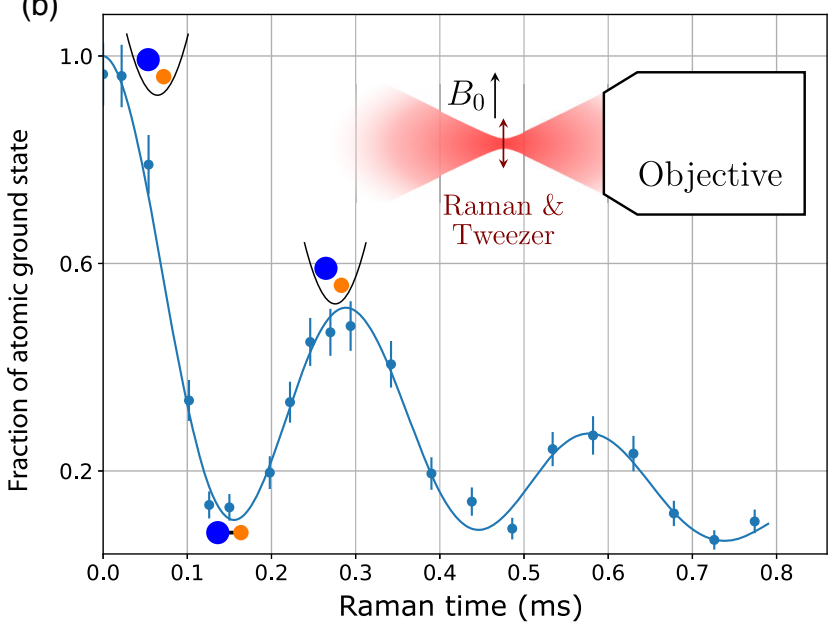

FIG. 3. Coherent transfer of atoms to molecules. The molecular state is dark to the imaging step and corresponds to zero signal. (a) Raman difference frequency scans for various durations showing the resonance as a function of the detuning from the fitted resonance at 770.5715(1) MHz, near the calculated value of 763 MHz. (b) Raman pulse-length scan on resonance. A decaying Rabi oscillation shows the coherence of the Raman transfer process. A model is fitted to (a) and (b) to determine the Raman Rabi frequency and loss rates. Inset: geometry and polarization of trap and Raman beam relative to the magnetic field. The $3.25 \mathrm{~mW}$ beam is focused to a waist of $0.9 \mu \mathrm{m}$ that confines the atoms and molecule. A perpendicular $B_{0}=8.83(2) \mathrm{G}$ magnetic field defines the quantization axis and the atoms experience predominantly $\pi$-polarized light. 
stability requirement to the percent level and enhances the Raman Rabi frequency.

Experimentally, we first prepare two atoms in a welldefined external and internal quantum state by using techniques developed previously [41,51,52]. In brief, the experimental cycle begins by stochastically loading a single ${ }^{23} \mathrm{Na}$ atom and a single ${ }^{133} \mathrm{Cs}$ atom into separate optical tweezers. The atoms are initially imaged to postselect loading of both atoms versus none or one atom. After imaging, we turn on a 8.83(2) G magnetic field to define the quantization axis for the state preparation and molecule formation steps. Raman sideband cooling then prepares both atoms simultaneously in the three-dimensional motional ground state of their optical tweezers, leaving the atoms in the spin state $\left|\uparrow_{\mathrm{Na}} \uparrow_{\mathrm{Cs}}\right\rangle \equiv\left|f=2, m_{f}=2\right\rangle_{\mathrm{Na}}\left|f=4, m_{f}=4\right\rangle_{\mathrm{Cs}}$, which has a small scattering length. The weak two-atom interaction allows merging of the two tweezers with minimum perturbation so that they remain in the motional ground state.

After merging the tweezers, we drive the atoms into spin combination $\left|\uparrow_{\mathrm{Na}} \downarrow_{\mathrm{Cs}}\right\rangle$ with a large scattering length by performing a Cs spin flip while taking into account the $-30.7 \mathrm{kHz}$ interaction shift [50]. This is the initial atomic state for Raman transfer. The spin flip selectively transfers atoms in the relative motional ground state, removing any background from atoms in excited states of relative motion [53]. For the experiment reported here, $31 \%$ of initial twoatom population is transferred. Of this population, over $60 \%$ is in the ground state of center-of-mass motion, inferred from Raman sideband thermometry.

To transfer the atom pair into the target weakly bound molecular state, we modulate the tweezer beam with a second frequency near $770 \mathrm{MHz}$, as shown in Fig. 2. The dual use of the tweezer beam for confinement and Raman transfer not only minimizes photon scattering, but also allows a tight focus to minimize the transfer duration. A tweezer frequency far detuned (by $-151 \mathrm{GHz}$ ) from $v^{\prime}=0 \quad(<50 \mathrm{MHz}$ natural linewidth $)$ reduces resonant scattering [41]. Furthermore, two filters, each with a linewidth (FWHM) of $50 \mathrm{GHz}$, clean the laser spectrum and prevent broadband noise from causing unwanted excitation. As shown in Fig. 2, one filter immediately follows the laser, while the second filter precedes the focusing objective for final cleanup of the laser spectrum.

Figure 3 shows a Fourier-limited resonance together with Rabi oscillations between the atomic and molecular states. A decaying Rabi oscillation with frequency $2 \pi \times 3.28(4) \mathrm{kHz}$ fitted to the data suggests that $69 \%$ of atoms are transferred into the molecular state after a $\pi$ pulse, with the majority of molecules in the ground state of motion [26,27].

To understand the details and limitations of the Raman transfer process better, we measured the properties of the two-photon resonance as a function of tweezer power and single-photon detuning. Known dependencies of the light shift and Raman Rabi frequency $\Omega_{R}$ on detuning $\Delta$ allow experimental determination of the Rabi frequencies $\Omega_{a}$ and $\Omega_{m}$ whose ratio critically affects the transfer efficiency. Both the light shift and $\Omega_{R}$ follow a $1 / \Delta$ slope as shown in Figs. 4(a) and 4(b) and include a constant offset that we attribute to coupling to other excited states that are further away in energy. The $1 / \Delta$ components due to the nearby $v^{\prime}=0$ intermediate state determine $\Omega_{m}$ and $\Omega_{a}$ in Table I.

Figure 4(c) shows the power dependence of $\Omega_{m}$ and $\Omega_{a}$, where $\Omega_{m}$ scales as $P^{1 / 2}$ as expected. As discussed previously, the scaling of $\Omega_{a}$ is $P^{7 / 8}$ for weakly interacting particles. However, due to the strong interaction between the two atoms in the $\left|\uparrow_{\mathrm{Na}} \downarrow_{\mathrm{Cs}}\right\rangle$ state, this approximation
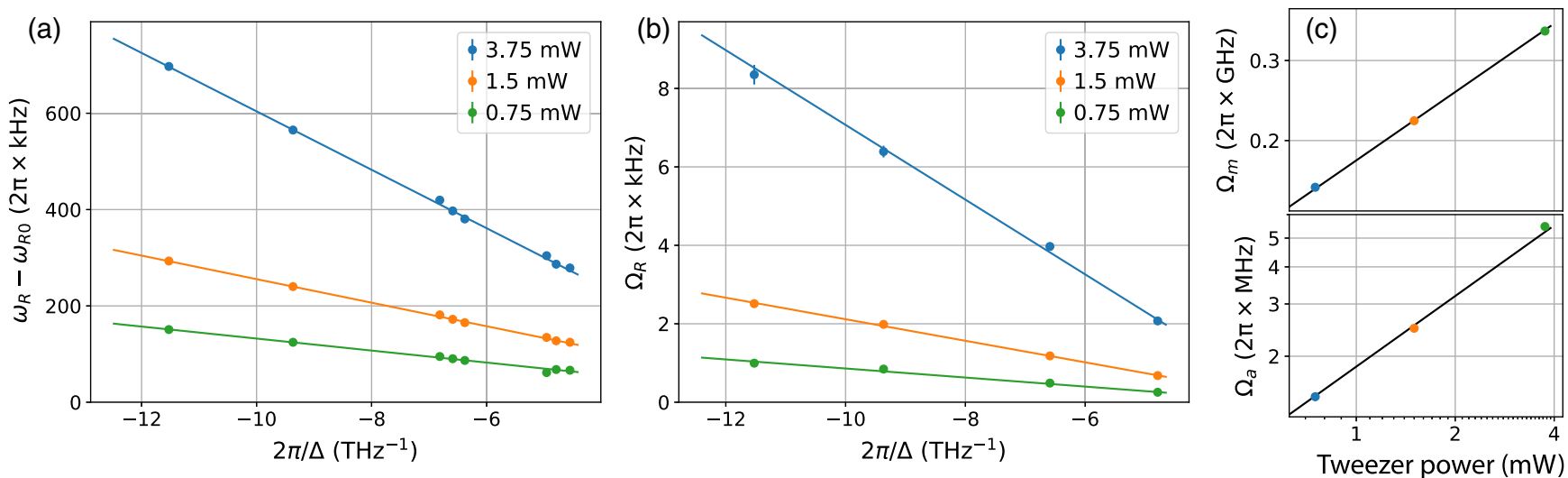

FIG. 4. Raman transition parameters as a function of tweezer power and detuning. (a) The Raman resonance $\omega_{R}$ fitted to $a_{P}+b_{P} / \Delta$, where $a_{P}$ and $b_{P}=\left(\Omega_{a}^{2}-\Omega_{m}^{2}\right) / 2$ are the power $(P)$ dependent background and $v^{\prime}=0$ contributions to the light shift and $\Delta \equiv 2 \pi \times$ $\left(f_{\mathrm{PA} 0}-f_{\text {tweezer }}\right)$ is the detuning from the $v^{\prime}=0$ resonance frequency $f_{\mathrm{PA} 0} \cdot a_{P}$ is fitted to a model including linear and small quadratic light shift to obtain the Raman resonance frequency at zero tweezer power $\omega_{R 0}=2 \pi \times 770.1969(2) \mathrm{MHz}$, where the statistical uncertainty is shown. (b) Raman Rabi frequency $\Omega_{R}$ fitted to $c_{P}+d_{P} / \Delta$, where $c_{P}$ and $d_{P}=\Omega_{a} \Omega_{m} / 2$ are the background and $v^{\prime}=0$ contributions that scale as $P^{1.29}$. The detuning is calculated from $f_{\mathrm{PA} 0}$ fitted in (a). Fit parameters are listed in Table I. (c) Tweezer power dependency of $\Omega_{m}$ (top) and $\Omega_{a}$ (bottom) calculated from $b_{P}$ and $d_{P}$ on a log-log scale showing approximate $P^{0.5}$ scaling of $\Omega_{m}$ and $P^{0.79}$ scaling of $\Omega_{a}$. 
TABLE I. Fitting results for Figs. 4(a) and 4(b). $\Omega_{R}$ is reported at $-151 \mathrm{GHz}$ detuning from the $v^{\prime}=0$ state. At $3.75 \mathrm{~mW}$, $\Omega_{m} / \Omega_{a}=0.016$, near the theory prediction of 0.013 . The measured Rabi rate $\Omega_{R}$ is only $63 \%$ of $\Omega_{m} \Omega_{a} /(2 \Delta)$ due to interference from further-detuned Raman processes.

\begin{tabular}{lccc}
\hline \hline$P(\mathrm{~mW})$ & 0.75 & 1.5 & 3.75 \\
\hline$f_{\mathrm{PA} 0}(\mathrm{GHz})$ & \multicolumn{3}{c}{288711.8} \\
\hline$a_{P}(2 \pi \times \mathrm{MHz})$ & $770.20452(6)$ & $770.2081(1)$ & $770.1943(3)$ \\
$b_{P}\left(4 \pi^{2} \times \mathrm{MHz} \cdot \mathrm{GHz}\right)$ & $-12.46(2)$ & $-24.44(3)$ & $-60.66(8)$ \\
\hline$c_{P}(2 \pi \times \mathrm{kHz})$ & $0.29(2)$ & $0.63(4)$ & $2.4(2)$ \\
$d_{P}\left(4 \pi^{2} \times \mathrm{MHz} \cdot \mathrm{GHz}\right)$ & $0.115(4)$ & $0.275(6)$ & $0.95(3)$ \\
\hline$\Omega_{R}(2 \pi \times \mathrm{kHz})$ & $0.49(2)$ & $1.18(3)$ & $3.97(9)$ \\
$\Omega_{m}(2 \pi \times \mathrm{MHz})$ & & & $348.3(3)$ \\
$\Omega_{a}(2 \pi \times \mathrm{kHz})$ & & & $5.5(2)$ \\
\hline \hline
\end{tabular}

breaks down. Coupled-channel calculations show that the wave function scaling is well approximated by $P^{0.29}$ within the range of confinement in our experiment and the expected scaling of $\Omega_{a} \propto P^{0.79}$ agrees with the data.

While the measured single-photon Rabi frequencies of Table I are in broad agreement with calculations (see Appendixes D and E), calculated scattering rates of the molecular and atomic state underestimate the decoherence in Fig. 3(b). From experimental measurements, the Raman transfer efficiency is limited by the molecular lifetime, together with a reduction in the Raman Rabi frequency due to destructive interference with intermediate states beyond $v^{\prime}=0$ (see Table I). We discuss loss and decoherence below.

The molecular lifetime measurement in Fig. 5(a) is performed by preparing the molecule with a $\pi$ pulse, followed after a variable delay by a second dissociating $\pi$ pulse. The measured lifetime o $0.196(14) \mathrm{ms}$ is consistent with the decaying timescale of 0.203(13) $\mathrm{ms}$ of the Rabi oscillation in Fig. 3(b). Preliminary experiments and theoretical considerations indicate that the molecular lifetime may be limited by two-photon coupling to the atomic continuum [54]. Atom loss is shown to be small in Fig. 5(b) by measuring the two-body lifetimes of the atoms directly without the second Raman frequency. In principle, destructive interference that reduces the Raman Rabi rate $\Omega_{R}$ for negative detunings $\Delta$ changes to constructive interference for positive detunings, but additional molecular resonances make the positive region unusable. More negative detunings that might reduce the scattering rate were prevented by vanishing of $\Omega_{R}$.

Separately we observe a decrease in coherence by a factor of 2 without laser spectrum filters, suggesting that spectral impurity of the laser can be a significant source of loss. While we have not fully characterized the sources of broadband noise, possibilities include amplified spontaneous emission from the laser and fiber nonlinearities.
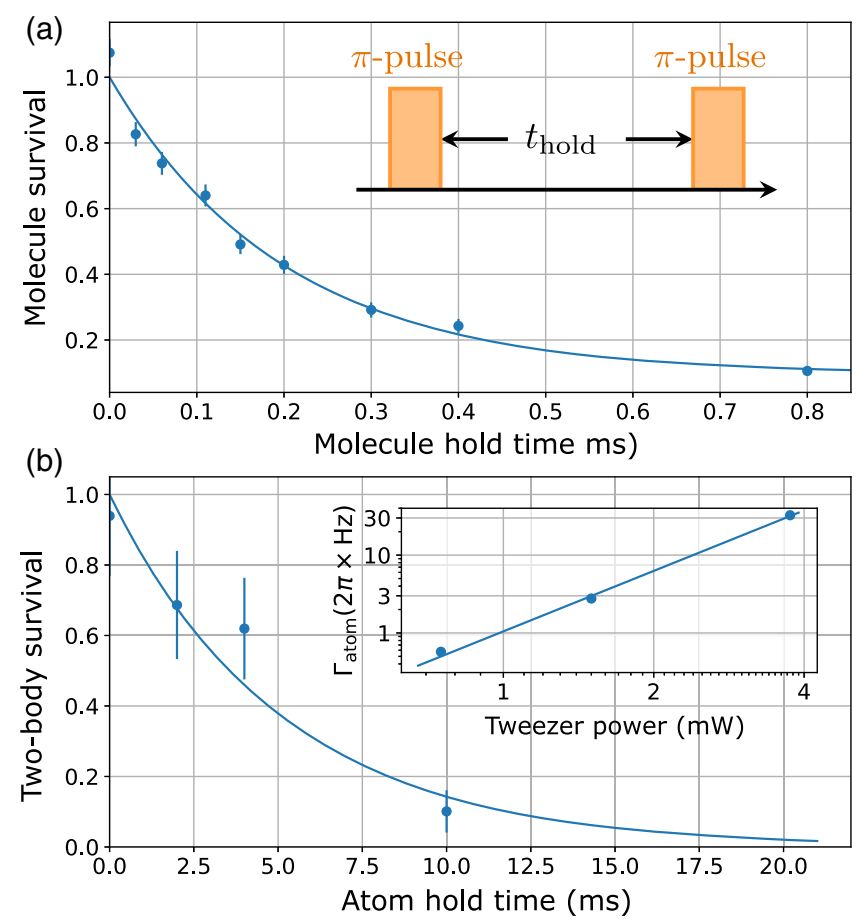

FIG. 5. Lifetime measurements with about $3.25 \mathrm{~mW}$ optical power. (a) Direct measurement of molecule lifetime. Molecule survival is detected by dissociating back to atoms via a second Raman transition. The lifetime is consistent with the decay of the Rabi oscillation in Fig. 3(b). Inset: pulse sequence for the lifetime measurement. (b) Two-body atom lifetime of 5(1) ms limited by off-resonance photoassociation. This is used to improve the fitting of the Raman transfer data. Inset: atomic scattering rate scales as $P_{\text {tweezer }}^{2.58} \times 2 \pi \times 1.05(6) \mathrm{Hz} / \mathrm{m} \mathrm{W}^{2.58}$ on a log-log scale; this is consistent with a two-photon scattering process.

Other potential decoherence sources include fluctuations of the tweezer intensity and magnetic field, although the shape of the Rabi oscillation in Fig. 3(b) indicates that loss rather than frequency fluctuations are responsible for decoherence. Based on the ratio between $\Omega_{R}$ and the light shift, the requirement on the tweezer intensity stability is $1 \%$ at $3.75 \mathrm{~mW}$ power. We stabilize the power to $0.1 \%$, indicating that in the absence of beam-waist fluctuations, light shift is not a major source of decoherence. Similarly, the measured Zeeman shift of $42.2(2) \mathrm{kHz} / \mathrm{G}$ does not cause significant decoherence for the measured magnetic field fluctuation of $1.5 \mathrm{mG}$.

In conclusion, we have coherently formed a weakly bound $\mathrm{NaCs}$ molecule in an optical tweezer by optical Raman transfer. This process is enabled by utilizing a deeply bound intermediate state, as well as highly interacting initial atomic states. A theoretical investigation including 8 excited molecular electronic potentials, the electronically excited atomic continuum, and coupled-channel ground-state wave functions indicates the potential for higher transfer efficiency than the observed value of $69 \%$. Future experiments may benefit from better balancing of the up-leg and downleg Rabi frequencies, for example, by driving to more deeply 
bound states. If possible, destructive interference that reduces the two-photon Rabi rate should be avoided. Nonlinear optical effects that limit the molecular state lifetime can also be explored.

Our technique can be applied to form a diverse set of molecular species, because it does not rely on a magnetic Feshbach resonance, states bound by only a few megahertz, or a narrow excited state. The formation of weakly bound molecules is a key step in forming rovibrational groundstate molecules. By scaling up to many optical tweezers [55-57], large arrays with arbitrary geometry of highly controlled molecules can be achieved. These molecules comprise a flexible platform for quantum simulation, quantum computing, precision measurements applications.

We thank Bo Gao and Paul Julienne for discussion and Robert Moszynski for providing theoretical transition dipole moments of NaCs. This work is supported by the NSF (PHY-1806595), the AFOSR (FA9550-19-1-0089), ARO DURIP (W911NF1810194), and the Arnold and Mabel Beckman foundation. J. T.Z. is supported by a National Defense Science and Engineering Graduate Fellowship. W. B.C. is supported by a Max PlanckHarvard Research Center for Quantum Optics fellowship. $\mathrm{K}$. W. is supported by an NSF GRFP fellowship. J. M. H. is supported by the UK Engineering and Physical Sciences Research Council (EPSRC) Grants No. EP/N007085/1, No. EP/P008275/1, and No. EP/P01058X/1. R. G.-F. acknowledges financial support of the Spanish Project FIS2017-89349-P (MINECO) and the Andalusian research group FQM-207.

\section{APPENDIX A: THREE-LEVEL RAMAN TRANSFER WITH CROSS-COUPLING}

In our system, the separation in energy between the initial and target state is much smaller than the singlephoton detuning $\Delta$ from the intermediate state; see Fig. 1(a) in the main text. Thus, there is significant cross-coupling for the scattering rate and light shift, where each state is coupled to the excited state by the power in both beams. Under these conditions, the scattering rate and light shift are proportional to the total power $P_{\text {tot }}=P_{m}+P_{a}$, where $P_{m}\left(P_{a}\right)$ is the power in the beam that addresses the molecular (atomic) state. The Raman Rabi frequency is proportional to $\sqrt{P_{m} P_{a}}$. With a fixed total power and thus fixed scattering rate, the Raman Rabi frequency is maximized when $P_{m}=P_{a}=P_{\text {tot }} / 2$.

For the purposes of this work, we denote $\Omega_{a}\left(\Omega_{m}\right)$ as the Rabi frequencies to drive a transition from the atom (target molecule) state to the intermediate state used in the Raman transition corresponding to the power in a single beam, $P_{a}$ $\left(P_{m}\right)$. Thus, the Raman Rabi frequency is $\Omega_{a} \Omega_{m} /(2 \Delta)$, where $\Delta$ is the single-photon detuning. Cross-coupling affects the scattering rate and light shift, and assuming equal powers in both beams, these quantities become
$\Gamma_{e} \Omega_{m}^{2} /\left(2 \Delta^{2}\right)+\Gamma_{e} \Omega_{a}^{2} /\left(2 \Delta^{2}\right)$ and $\Omega_{m}^{2} /(2 \Delta)-\Omega_{a}^{2} /(2 \Delta)$, where $\Gamma_{e}$ is the excited-state linewidth. Since $\Omega_{m} \gg \Omega_{a}$, the scattering rate and light shift can be approximated as $\Gamma_{e} \Omega_{m}^{2} /\left(2 \Delta^{2}\right)$ and $\Omega_{m}^{2} /(2 \Delta)$.

\section{APPENDIX B: RAMAN TRANSFER WITH MANY INTERMEDIATE STATES}

With multiple intermediate states, the total Raman Rabi frequency, scattering rate, and light shift become a sum over all these possible states. In our model, we consider all vibrational states of $c^{3} \Sigma^{+}(\Omega=1)$ and states in the atomic continuum as intermediate states. The Rabi frequency between two states is proportional to the electronic dipole moment, arising from the electronic wave functions, and the overlap of the vibrational wave functions. Under the assumption that the electronic dipole moment $d$ is the same for all transitions between the ground state and the excited electronic state, the total Raman Rabi frequency becomes

$$
\Omega_{R}=\frac{d^{2} E^{2}}{2 \hbar^{2}} \sum_{v^{\prime}} \frac{\left\langle a \mid v^{\prime}\right\rangle\left\langle v^{\prime} \mid m\right\rangle}{\Delta_{v^{\prime}}},
$$

where $|a\rangle(|m\rangle)$ are the relative coordinate wave functions for the trapped atomic (target molecular) state, and $\left|v^{\prime}\right\rangle$ is the relative-coordinate wave function for the excited state which includes both bound molecular states and the atomic continuum. $\Delta_{v^{\prime}}$ is a single-photon detuning with respect to the $\left|v^{\prime}\right\rangle$ state, and $E$ is the electric field for the power in the single beam given a waist size. We can divide this sum into a sum over all the bound molecular states, and a sum over atomic continuum states,

$$
\begin{aligned}
\Omega_{R}= & \frac{d^{2} E^{2}}{2 \hbar^{2}} \sum_{v^{\prime} \leq v_{\max }^{\prime}} \frac{\left\langle a \mid v^{\prime}\right\rangle\left\langle v^{\prime} \mid m\right\rangle}{\Delta_{v^{\prime}}} \\
& +\frac{d^{2} E^{2}}{2 \hbar^{2}} \sum_{v^{\prime}>v_{\max }^{\prime}} \frac{\left\langle a \mid v^{\prime}\right\rangle\left\langle v^{\prime} \mid m\right\rangle}{\Delta_{v^{\prime}}},
\end{aligned}
$$

where $v_{\max }^{\prime}$ corresponds to the excited molecular state, which is most weakly bound. The first term can be calculated numerically given an excited-state potential. To calculate the second term, we first make an assumption that we are far enough detuned from the contributing continuum states that they are all at approximately the same detuning, $\Delta_{\text {thresh }}$. This allows us to pull it out of the sum, and we obtain

$$
\begin{aligned}
\Omega_{R} \approx & \frac{d^{2} E^{2}}{2 \hbar^{2}} \sum_{v^{\prime} \leq v_{\max }^{\prime}} \frac{\left\langle a \mid v^{\prime}\right\rangle\left\langle v^{\prime} \mid m\right\rangle}{\Delta_{v^{\prime}}} \\
& +\frac{d^{2} E^{2}}{2 \hbar^{2} \Delta_{\text {thresh }}} \sum_{v^{\prime}>v_{\max }^{\prime}}\left\langle a \mid v^{\prime}\right\rangle\left\langle v^{\prime} \mid m\right\rangle .
\end{aligned}
$$

From requiring completeness and orthogonality, 


$$
\sum_{v^{\prime}}\left\langle a \mid v^{\prime}\right\rangle\left\langle v^{\prime} \mid m\right\rangle=\langle a \mid m\rangle=0
$$

we derive an expression for the second sum in Eq. (B3),

$$
\sum_{v^{\prime}>v_{\max }^{\prime}}\left\langle a \mid v^{\prime}\right\rangle\left\langle v^{\prime} \mid m\right\rangle=-\sum_{v^{\prime} \leq v_{\max }^{\prime}}\left\langle a \mid v^{\prime}\right\rangle\left\langle v^{\prime} \mid m\right\rangle,
$$

which can be calculated from the vibrational states of the excited-state potential. Thus, the Raman Rabi frequency can be approximated as

$$
\begin{aligned}
\Omega_{R} \approx & \frac{d^{2} E^{2}}{2 \hbar^{2}} \sum_{v^{\prime} \leq v_{\max }^{\prime}} \frac{\left\langle a \mid v^{\prime}\right\rangle\left\langle v^{\prime} \mid m\right\rangle}{\Delta_{v^{\prime}}} \\
& -\frac{d^{2} E^{2}}{2 \hbar^{2} \Delta_{\text {thresh }}} \sum_{v^{\prime} \leq v_{\max }^{\prime}}\left\langle a \mid v^{\prime}\right\rangle\left\langle v^{\prime} \mid m\right\rangle .
\end{aligned}
$$

We can account for the atomic continuum in the scattering rate in a similar way,

$$
\begin{aligned}
\Gamma_{s} \approx & \frac{d^{2} E^{2}}{2 \hbar^{2}} \sum_{v^{\prime} \leq v_{\max }^{\prime}} \Gamma_{v^{\prime}} \frac{\left\langle m \mid v^{\prime}\right\rangle\left\langle v^{\prime} \mid m\right\rangle}{\Delta_{v^{\prime}}^{2}} \\
& +\frac{d^{2} E^{2} \Gamma}{2 \hbar^{2} \Delta_{\text {thresh }}^{2}} \sum_{v^{\prime}>v_{\max }^{\prime}}\left\langle m \mid v^{\prime}\right\rangle\left\langle v^{\prime} \mid m\right\rangle,
\end{aligned}
$$

where $\Gamma_{v^{\prime}}$ is the excited-state linewidth for the $\left|v^{\prime}\right\rangle$ state, and we have assumed the atomic continuum states all have the same linewidth $\Gamma$. Using normalization, we derive an expression for the sum in the second term:

$$
\sum_{v^{\prime}>v_{\max }^{\prime}}\left\langle m \mid v^{\prime}\right\rangle\left\langle v^{\prime} \mid m\right\rangle=1-\sum_{v^{\prime} \leq v_{\max }^{\prime}}\left\langle m \mid v^{\prime}\right\rangle\left\langle v^{\prime} \mid m\right\rangle .
$$

We then arrive at an expression for the scattering rate, accounting for the atomic continuum:

$$
\begin{aligned}
\Gamma_{s} \approx & \frac{d^{2} E^{2}}{2 \hbar^{2}} \sum_{v^{\prime} \leq v_{\max }^{\prime}} \Gamma_{v^{\prime}} \frac{\left\langle m \mid v^{\prime}\right\rangle\left\langle v^{\prime} \mid m\right\rangle}{\Delta_{v^{\prime}}^{2}} \\
& +\frac{d^{2} E^{2} \Gamma}{2 \hbar^{2} \Delta_{\text {thresh }}^{2}}\left(1-\sum_{v^{\prime} \leq v_{\max }^{\prime}}\left\langle m \mid v^{\prime}\right\rangle\left\langle v^{\prime} \mid m\right\rangle\right) .
\end{aligned}
$$

We have replaced the full continuum with an effective state at the atomic threshold with strength proportional to the amount of wave function overlap between the target molecular state and all atomic continuum states. This model leads to a component in the scattering rate that is proportional to $1 / \Delta_{\text {thresh }}^{2}$ and is smaller as one detunes farther from the threshold. We note that the first term can also scale nearly as $1 / \Delta_{\text {thresh }}^{2}$, if, as in our case, the largest terms in the summation are from states near the threshold.
A very similar result holds for the light shift $\delta$ and is written here for completeness:

$$
\begin{aligned}
\delta= & \frac{d^{2} E^{2}}{2 \hbar^{2}} \sum_{v^{\prime} \leq v_{\max }^{\prime}} \frac{\left\langle m \mid v^{\prime}\right\rangle\left\langle v^{\prime} \mid m\right\rangle}{\Delta_{v^{\prime}}} \\
& +\frac{d^{2} E^{2}}{2 \hbar^{2} \Delta_{\text {thresh }}}\left(1-\sum_{v^{\prime} \leq v_{\max }^{\prime}}\left\langle m \mid v^{\prime}\right\rangle\left\langle v^{\prime} m\right\rangle\right) .
\end{aligned}
$$

\section{APPENDIX C: COMPUTATIONAL METHODS FOR BOUND AND SCATTERING STATES}

For scattering and near-threshold bound states, we solve the Schrödinger equation by coupled-channel methods [58], using a basis set in which the electron spins $s_{\mathrm{Na}}$ and $s_{\mathrm{Cs}}$ and the nuclear spins $i_{\mathrm{Na}}$ and $i_{\mathrm{Cs}}$ are separately coupled:

$$
\left|\left(s_{\mathrm{Na}} s_{\mathrm{Cs}} S\right)\left(i_{\mathrm{Na}} i_{\mathrm{Cs}} I\right) F M_{F}\right\rangle .
$$

Here $S$ and $I$ are the total electron and nuclear spins of the molecule or atomic pair. Their resultant $F$ is the total spin, with projection $M_{F}$ onto the axis of the magnetic field. In principle, $F$ can be further coupled to the rotational angular momentum $L$, but in the present work only functions with $L=0$ are included. In the absence of a magnetic field, $F$ and $M_{F}$ are both good quantum numbers, but in a magnetic field, only $M_{F}$ is conserved. The calculations in this paper use basis sets with all possible values of $S, I$, and $F$ (for the required value of $M_{F}$ ) that can be constructed with $s_{\mathrm{Na}}=s_{\mathrm{Cs}}=1 / 2, i_{\mathrm{Na}}=3 / 2$, and $i_{\mathrm{Cs}}=7 / 2$.

Scattering calculations are carried out using the MOLSCAT package $[59,60]$ and bound-state calculations using the related BOUND package $[60,61]$. The calculations use singlet and triplet interaction potentials for $\mathrm{Na}+\mathrm{Cs}$ obtained by adjusting the potentials of Docenko et al. [62] at short range, using the same procedure as for $\mathrm{KCs}$ [63], to reproduce the experimental binding energy of the least-bound triplet state [50] and the position of the $s$-wave Feshbach resonance near $864 \mathrm{G}$ [26]. The atomic nuclear hyperfine coupling and Zeeman effects are fully included, using the Hamiltonian described in Ref. [64].

The bound-state calculations were initially used to predict the binding energies of states that lie within about $1 \mathrm{GHz}$ of each atomic threshold of interest. At zero magnetic field, the states fall into groups from each hyperfine state of the atomic pair, labeled by $\left(f_{\mathrm{Na}}, f_{\mathrm{Cs}}\right)$. For example, for the $\left(f_{\mathrm{Na}}, f_{\mathrm{Cs}}\right)=(2,3)$ states that are of principal interest here, each group comprises 5 zero-field levels with $F=1,2,3,4$, and 5; one such group is spread out over a range of energies between 290 and $770 \mathrm{MHz}$ below the $\left(f_{\mathrm{Na}}, f_{\mathrm{Cs}}\right)=(2,3)$ threshold. In a magnetic field, 
each zero-field level splits into $2 F+1$ Zeeman sublevels labeled by $M_{F}$. For each sublevel we can calculate the dependence of the energy on magnetic field, in order to choose a target state for which the Raman transition frequency depends only weakly on field.

The state that we ultimately chose as the target state for Raman transfer is the least-bound $\left(F, M_{F}\right)=(5,5)$ sublevel with $\left(f_{\mathrm{Na}}, f_{\mathrm{Cs}}\right)=(2,3)$, which was predicted at a binding energy $763 \mathrm{MHz}$ below the $\left(f_{\mathrm{Na}}, f_{\mathrm{Cs}}\right)=(2,3)$ threshold. As described in the text, it was subsequently located experimentally at a binding energy of 770.1969(2) $\mathrm{MHz}$ at 8.83(2) G.

Multichannel bound-state wave functions, with components labeled by $S, I$, and $F$, are calculated using the method of Thornley and Hutson [65]. For molecular bound states, they are obtained on a grid of internuclear distances from 2.4 to $50 \AA$. For states of confined atomic pairs, a harmonic confining potential is added to the molecular potentials and the grid is extended to $3000 \AA$.

\section{APPENDIX D: EXCITED MOLECULAR STATES}

To obtain a full calculation for the expected Raman Rabi frequency, light shift, and scattering, we need to account for all the excited molecular states. The lowest eight excited molecular potentials in $\mathrm{NaCs}$ asymptote to the dissociation limit of $\mathrm{Na} 3 S+\mathrm{Cs} 6 P$. These eight potentials can be written in the Hund's case (a) basis as $c^{3} \Sigma_{\Omega=0^{-}}^{+}, c^{3} \Sigma_{\Omega=1}^{+}, b^{3} \Pi_{\Omega=0^{-}}, b^{3} \Pi_{\Omega=0^{+}}, b^{3} \Pi_{\Omega=1}, b^{3} \Pi_{\Omega=2}$, $B^{1} \Pi_{\Omega=1}, A^{1} \Sigma_{\Omega=0^{+}}^{+}[43]$. Because of spin-orbit coupling, especially large in Cs, the excited molecular states are mixed, containing components in multiple potentials. Fortunately, single-channel potential energy curves for $c^{3} \Sigma_{1}^{+}$and $B^{1} \Pi_{1}$ have been experimentally determined, and are predictive across the entire energy range of the potential $[44,46,48]$. Since there is only one $\Omega=2$ potential at this dissociation limit, a single-channel potential has been determined for $b^{3} \Pi_{2}$ as well [47]. Unfortunately, single-channel potentials for the other electronic states do not exist. A deperturbation analysis was required for states belonging to the mixed $A-b$ complex, and the fitted potentials and spin-orbit coupling coefficients are only accurate within the energy range of the measured experimental data [45]. Since our experiment attempts to work in a regime where the predominant Raman coupling comes from the $v^{\prime}=0$ state of $c^{3} \Sigma_{1}^{+}$, the contribution of the other seven potentials is mainly to a background that is nearly detuning independent. Thus, for the computational ease, we use the diabatic potentials for $b^{3} \Pi_{0}, b^{3} \Pi_{1}$, and $A^{1} \Sigma_{0^{+}}^{+}$. Lastly, to our knowledge, no potential fit to experimental data exists for the $c^{3} \Sigma_{0^{-}}^{+}$state. Assuming that its contribution is only to the background, as an approximation, we simply double the contribution of all states except $v^{\prime}=0$ from $c^{3} \Sigma_{1}^{+}$.

\section{APPENDIX E: CALCULATING MATRIX ELEMENTS WITH COUPLED-CHANNEL GROUND STATE}

To calculate Rabi frequencies for transitions to the eight excited molecular potentials, we obtain their wave functions from a single-channel calculation [66] using the potential energy curves described in Appendix D. We then calculate overlap integrals between these functions and either the triplet $(S=1)$ or singlet $(S=0)$ components of the coupled-channel wave functions for either the target molecular state or the confined atomic state. These calculations assume that the intermediate states have pure singlet or triplet character and neglect its nuclear spin character, which is unknown. These calculations include theoretical electronic transition dipole moments, shown in Fig. 6, calculated with the same $a b$ initio methods as described in Ref. [67] for $\mathrm{Rb}_{2}$. Using previously calculated $\mathrm{NaCs}$ transition dipole moments [68,69] would lead to similar qualitative results.

The full calculation of the Raman Rabi frequency and scattering rate using the methods in Appendix B is shown in Fig. 7. The strong contribution from the atomic threshold arises from the large overlap to the near-threshold bound states shown in Fig. 8 for the $c^{3} \Sigma_{1}^{+}$state. This leads to a $1 / \Delta_{\text {thresh }}^{2}$ dependence of the scattering rate and favors using deeply bound states as the intermediate state for the Raman transfer. A similar result holds for the other excited-state potentials.

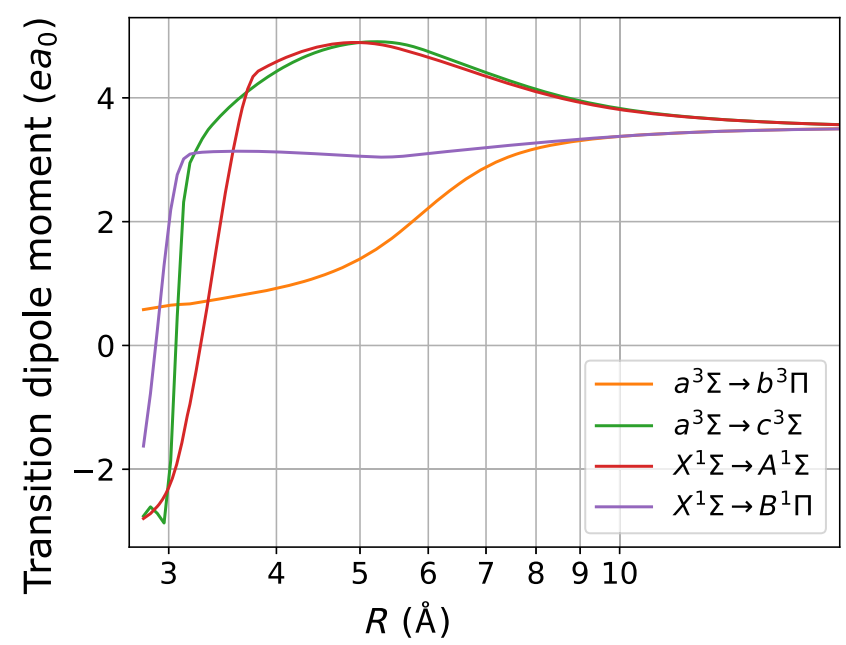

FIG. 6. Electronic transition dipole moments calculated via $a b$ initio methods between different electronic states of $\mathrm{NaCs}$. These transition dipole moments are shown in units of $e a_{0}$, where $e$ is the elementary charge and $a_{0}$ is the Bohr radius. They are plotted as a function of internuclear distance $R$. 


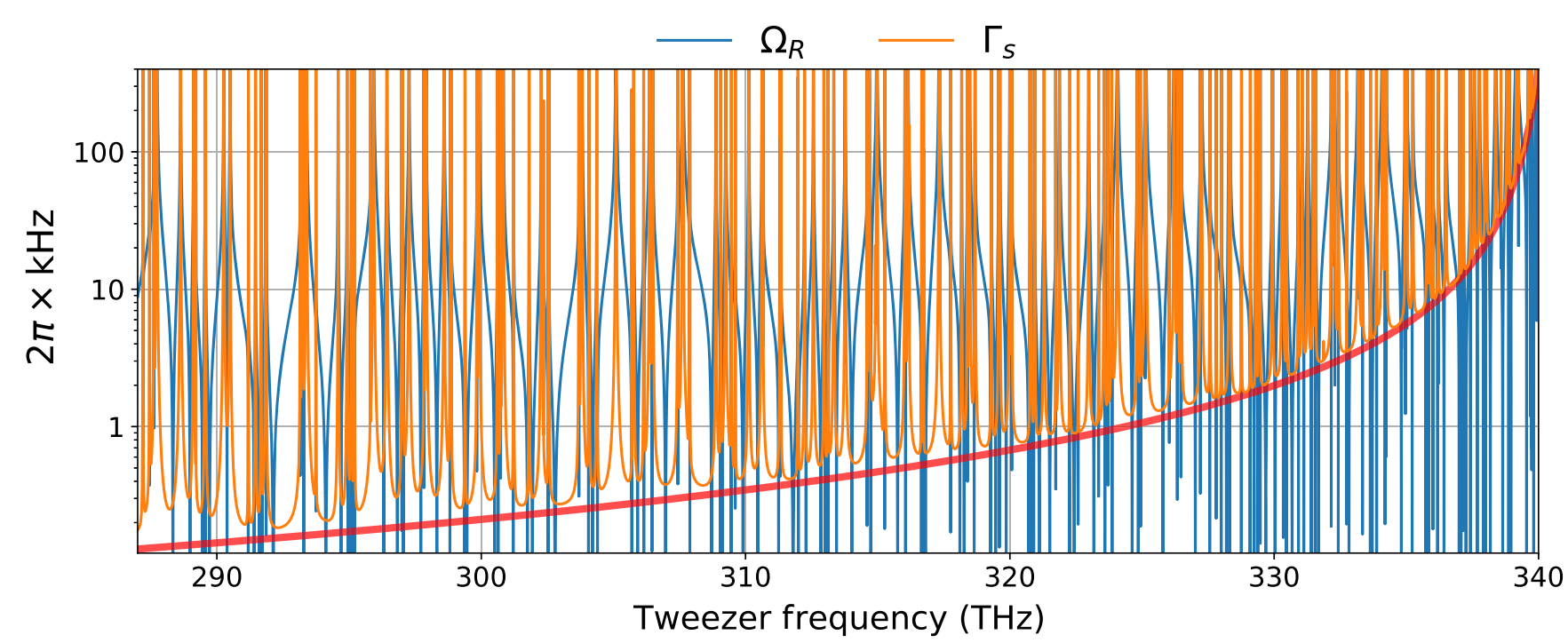

FIG. 7. Full calculation of the Raman Rabi frequency and scattering rate as a function of the single-photon frequency including all vibrational states of $c^{3} \Sigma^{+}(\Omega=1)$ and the atomic continuum. The second frequency in the Raman transition is offset by about $770 \mathrm{MHz}$ and resonant with the two-photon transition. The assumed excited-state linewidth for all molecular lines is $50 \mathrm{MHz}$. The red line shows the contribution that comes only from the near-threshold states.

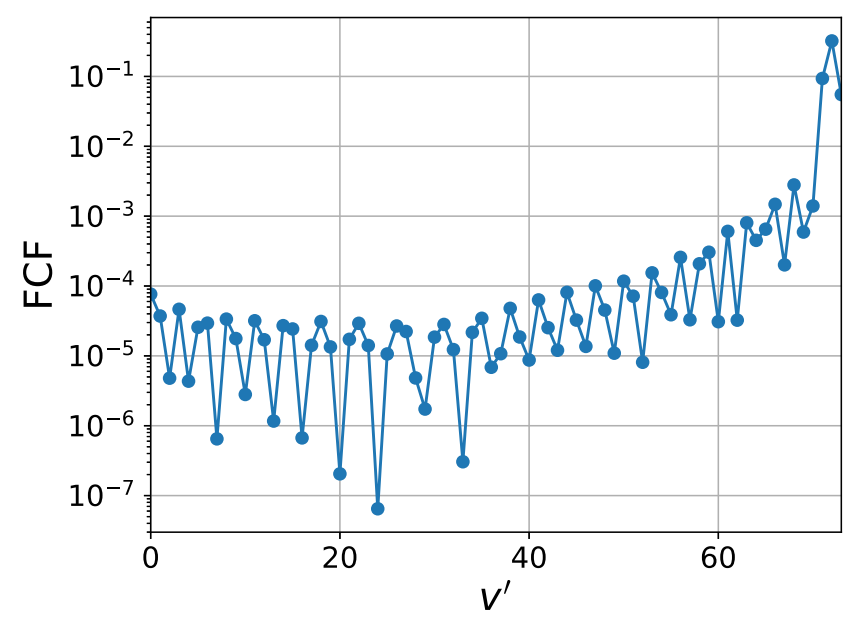

FIG. 8. The Franck-Condon factor (FCF) between the target molecular state and vibrational state $v^{\prime}$ of $c^{3} \Sigma_{1}^{+}$. The potential supports 74 bound states, and the predominant contribution arises from the near-threshold bound states. The total overlap with all the bound states is 0.4822 , which is $96.8 \%$ of the total triplet fraction of the target molecular state.

\section{APPENDIX F: EFFECTIVE MATRIX ELEMENTS TO A SINGLE STATE}

In this calculation, there is a matrix element, $\Omega_{a i j}, \Omega_{m i j}$ between each channel $i$ of the coupled-channel ground state and an excited state $j$. The total Raman Rabi frequency $\Omega_{R}$, light shift $\delta$, and off-resonant scattering $\Gamma_{s}$ contribution from this single excited state is calculated as

$$
\Omega_{R j}=\sum_{i} \frac{\Omega_{a i j} \Omega_{m i j}}{2 \Delta}
$$

$$
\begin{gathered}
\delta_{j}=\sum_{i} \frac{\Omega_{a i j}^{2}-\Omega_{m i j}^{2}}{2 \Delta}, \\
\Gamma_{s j}=\sum_{i} \Gamma_{e} \frac{\Omega_{a i j}^{2}+\Omega_{m i j}^{2}}{2 \Delta^{2}},
\end{gathered}
$$

where $\Delta$ is the single-photon detuning and $\Gamma_{e}$ is the excited-state linewidth. The experiment, through measuring the detuning dependence of the Raman Rabi frequency and the resonance frequency, extracts a single $\Omega_{m}$ and $\Omega_{a}$. In order to compare to the theory, we introduce effective $\Omega_{a}^{\prime}, \Omega_{m}^{\prime}, \Gamma_{e}^{\prime}$, so that $\Omega_{R}=\Omega_{a}^{\prime} \Omega_{m}^{\prime} /(2 \Delta), \delta=$ $\left(\Omega_{a}^{\prime 2}-\Omega_{m}^{\prime 2}\right) /(2 \Delta)$, and $\Gamma_{s}=\Gamma_{e}^{\prime}\left(\Omega_{a}^{\prime 2}+\Omega_{m}^{\prime 2}\right) /\left(2 \Delta^{2}\right)$. The effective Rabi frequencies for the $v^{\prime}=0$ state of $c^{3} \Sigma_{1}^{+}$ and their ratio are reported in Table II at $3.75 \mathrm{~mW}$ of tweezer power.

\section{APPENDIX G: SCALING OF ATOMIC MATRIX ELEMENT WITH TWEEZER POWER}

Since our coupled-channel calculations include the harmonic confinement, we can calculate the enhancement in $\Omega_{a}$ with increasing confinement. Using two calculations with $\omega_{\text {trap }}=36.5 \mathrm{kHz}$ and $\omega_{\text {trap }}=80 \mathrm{kHz}$ trapping frequencies, and assuming a power-law scaling, we find

TABLE II. The effective Rabi frequencies for the $v^{\prime}=0$ state of $c^{3} \Sigma_{1}^{+}$at $3.75 \mathrm{~mW}$ of tweezer power.

\begin{tabular}{lc}
\hline \hline$\Omega_{m}^{\prime}$ & $2 \pi \times 573 \mathrm{MHz}$ \\
$\Omega_{a}^{\prime}$ & $2 \pi \times 7.37 \mathrm{MHz}$ \\
$\Omega_{a}^{\prime} / \Omega_{m}^{\prime}$ & 0.013 \\
\hline \hline
\end{tabular}


that the wave function overlap scales as $\omega_{\text {trap }}^{0.58}=P^{0.29}$. Thus, $\Omega_{a} \propto P^{0.79}$, which was also experimentally verified. However, we note that in the actual experiment, our system does not have spherically symmetric confinement. The radial trapping frequency is about 5.7 times larger than the axial trapping frequency.

[1] S. S. Kondov, C.-H. Lee, K. H. Leung, C. Liedl, I. Majewska, R. Moszynski, and T. Zelevinsky, Molecular Lattice Clock with Long Vibrational Coherence, Nat. Phys. 15, 1118 (2019).

[2] I. Kozyryev and N. R. Hutzler, Precision Measurement of Time-Reversal Symmetry Violation with Laser-Cooled Polyatomic Molecules, Phys. Rev. Lett. 119, 133002 (2017).

[3] V. V. Flambaum and V. A. Dzuba, Electric Dipole Moments of Atoms and Molecules Produced by Enhanced Nuclear Schiff Moments, Phys. Rev. A 101, 042504 (2020).

[4] V. Andreev et al. (ACME Collaboration), Improved Limit on the Electric Dipole Moment of the Electron, Nature (London) 562, 355 (2018).

[5] W. B. Cairncross, D. N. Gresh, M. Grau, K. C. Cossel, T. S. Roussy, Y. Ni, Y. Zhou, J. Ye, and E. A. Cornell, Precision Measurement of the Electron's Electric Dipole Moment Using Trapped Molecular Ions, Phys. Rev. Lett. 119, 153001 (2017).

[6] J. J. Hudson, D. M. Kara, I. J. Smallman, B. E. Sauer, M. R. Tarbutt, and E. A. Hinds, Improved Measurement of the Shape of the Electron, Nature (London) 473, 493 (2011).

[7] A. Micheli, G. Brennen, and P. Zoller, A Toolbox for Lattice-Spin Models with Polar Molecules, Nat. Phys. 2, 341 (2006).

[8] N. Y. Yao, M. P. Zaletel, D. M. Stamper-Kurn, and A. Vishwanath, A Quantum Dipolar Spin Liquid, Nat. Phys. 14, 405 (2018).

[9] M. L. Wall, K. R. A. Hazzard, and A. M. Rey, From Atomic to Mesoscale: The Role of Quantum Coherence in Systems of Various Complexities (World Scientific, Singapore, 2015), ch. 1, pp. 3-38.

[10] M. Wall, K. Maeda, and L. D. Carr, Realizing Unconventional Quantum Magnetism with Symmetric Top Molecules, New J. Phys. 17, 025001 (2015).

[11] D. DeMille, Quantum Computation with Trapped Polar Molecules, Phys. Rev. Lett. 88, 067901 (2002).

[12] K.-K. Ni, T. Rosenband, and D. D. Grimes, Dipolar Exchange Quantum Logic Gate with Polar Molecules, Chem. Sci. 9, 6830 (2018).

[13] E. R. Hudson and W. C. Campbell, Dipolar Quantum Logic for Freely Rotating Trapped Molecular Ions, Phys. Rev. A 98, 040302(R) (2018).

[14] Y. Lin, D. R. Leibrandt, D. Leibfried, and C.-W. Chou, Quantum Entanglement between an Atom and a Molecule, Nature (London) 581, 273 (2020).

[15] N. Balakrishnan, Perspective: Ultracold Molecules and the Dawn of Cold Controlled Chemistry, J. Chem. Phys. 145, 150901 (2016).

[16] M.-G. Hu, Y. Liu, D. D. Grimes, Y.-W. Lin, A. H. Gheorghe, R. Vexiau, N. Bouloufa-Maafa, O. Dulieu, T. Rosenband, and K.-K. Ni, Direct Observation of Bimolecular Reactions of Ultracold KRb Molecules, Science 366, 1111 (2019).

[17] Y. Segev, M. Pitzer, M. Karpov, N. Akerman, J. Narevicius, and E. Narevicius, Collisions between Cold Molecules in a Superconducting Magnetic Trap, Nature (London) 572, 189 (2019).

[18] T. de Jongh, M. Besemer, Q. Shuai, T. Karman, A. van der Avoird, G. C. Groenenboom, and S. Y. T. van de Meerakker, Imaging the Onset of the Resonance Regime in Low-Energy NO-He Collisions, Science 368, 626 (2020).

[19] E. B. Norrgard, D. J. McCarron, M. H. Steinecker, M. R. Tarbutt, and D. DeMille, Submillikelvin Dipolar Molecules in a Radio-Frequency Magneto-optical Trap, Phys. Rev. Lett. 116, 063004 (2016).

[20] A. Prehn, M. Ibrügger, R. Glöckner, G. Rempe, and M. Zeppenfeld, Optoelectrical Cooling of Polar Molecules to Submillikelvin Temperatures, Phys. Rev. Lett. 116, 063005 (2016).

[21] S. Truppe, H. J. Williams, M. Hambach, L. Caldwell, N. J. Fitch, E. A. Hinds, B. E. Sauer, and M. R. Tarbutt, Molecules Cooled Below the Doppler Limit, Nat. Phys. 13, 1173 (2017).

[22] L. Anderegg, B. L. Augenbraun, Y. Bao, S. Burchesky, L. W. Cheuk, W. Ketterle, and J. M. Doyle, Laser Cooling of Optically Trapped Molecules, Nat. Phys. 14, 890 (2018).

[23] S. Ding, Y. Wu, I. A. Finneran, J. J. Burau, and J. Ye, Sub-Doppler Cooling and Compressed Trapping of $\mathrm{YO}$ Molecules at $\mu \mathrm{K}$ Temperatures, Phys. Rev. X 10, 021049 (2020).

[24] D. Mitra, N. B. Vilas, C. Hallas, L. Anderegg, B. L. Augenbraun, L. Baum, C. Miller, S. Raval, and J. M. Doyle, Direct Laser Cooling of a Symmetric Top Molecule, Science 369, 1366 (2020).

[25] L. De Marco, G. Valtolina, K. Matsuda, W. G. Tobias, J. P. Covey, and J. Ye, A Degenerate Fermi Gas of Polar Molecules, Science 363, 853 (2019).

[26] J. T. Zhang, Y. Yu, W. B. Cairncross, K. Wang, L. R. B. Picard, J. D. Hood, Y.-W. Lin, J. M. Hutson, and K.-K. Ni, Forming a Single Molecule by Magnetoassociation in an Optical Tweezer, Phys. Rev. Lett. 124, 253401 (2020).

[27] X. He, K. Wang, J. Zhuang, P. Xu, X. Gao, R. Guo, C. Sheng, M. Liu, J. Wang, J. Li, G. V. Shlyapnikov, and M. Zhan, Coherently Forming a Single Molecule in an Optical Trap, Science 370, 331 (2020).

[28] J. G. Danzl, E. Haller, M. Gustavsson, M. J. Mark, R. Hart, N. Bouloufa, O. Dulieu, H. Ritsch, and H.-C. Nägerl, Quantum Gas of Deeply Bound Ground State Molecules, Science 321, 1062 (2008).

[29] K.-K. Ni, S. Ospelkaus, M. H. G. de Miranda, A. Pe'er, B. Neyenhuis, J. J. Zirbel, S. Kotochigova, P. S. Julienne, D. S. Jin, and J. Ye, A High Phase-Space-Density Gas of Polar Molecules, Science 322, 231 (2008).

[30] F. Lang, K. Winkler, C. Strauss, R. Grimm, and J. H. Denschlag, Ultracold Triplet Molecules in the Rovibrational Ground State, Phys. Rev. Lett. 101, 133005 (2008).

[31] T. Takekoshi, L. Reichsöllner, A. Schindewolf, J. M. Hutson, C. R. Le Sueur, O. Dulieu, F. Ferlaino, R. Grimm, and H.-C. Nägerl, Ultracold Dense Samples of Dipolar RbCs Molecules in the Rovibrational and Hyperfine Ground State, Phys. Rev. Lett. 113, 205301 (2014). 
[32] P. K. Molony, P. D. Gregory, Z. Ji, B. Lu, M. P. Köppinger, C. R. Le Sueur, C. L. Blackley, J. M. Hutson, and S. L. Cornish, Creation of Ultracold ${ }^{87} \mathrm{Rb}^{133} \mathrm{Cs}$ Molecules in the Rovibrational Ground State, Phys. Rev. Lett. 113, 255301 (2014).

[33] J. W. Park, S. A. Will, and M. W. Zwierlein, Ultracold Dipolar Gas of Fermionic ${ }^{23} \mathrm{Na}^{40} \mathrm{~K}$ Molecules in Their Absolute Ground State, Phys. Rev. Lett. 114, 205302 (2015).

[34] M. Guo, B. Zhu, B. Lu, X. Ye, F. Wang, R. Vexiau, N. Bouloufa-Maafa, G. Quéméner, O. Dulieu, and D. Wang, Creation of an Ultracold Gas of Ground-State Dipolar ${ }^{23} \mathrm{Na}^{87} \mathrm{Rb}$ Molecules, Phys. Rev. Lett. 116, 205303 (2016).

[35] K. K. Voges, P. Gersema, M. M. zum Alten Borgloh, T. A. Schulze, T. Hartmann, A. Zenesini, and S. Ospelkaus, Ultracold Gas of Bosonic ${ }^{23} \mathrm{Na}^{39} \mathrm{~K}$ Ground-State Molecules, Phys. Rev. Lett. 125, 083401 (2020).

[36] S. Stellmer, B. Pasquiou, R. Grimm, and F. Schreck, Creation of Ultracold $\mathrm{Sr}_{2}$ Molecules in the Electronic Ground State, Phys. Rev. Lett. 109, 115302 (2012).

[37] G. Reinaudi, C. B. Osborn, M. McDonald, S. Kotochigova, and T. Zelevinsky, Optical Production of Stable Ultracold ${ }^{88} \mathrm{Sr}_{2}$ Molecules, Phys. Rev. Lett. 109, 115303 (2012).

[38] A. Green, H. Li, J. H. See Toh, X. Tang, K. C. McCormick, M. Li, E. Tiesinga, S. Kotochigova, and S. Gupta, Feshbach Resonances in p-Wave Three-Body Recombination within Fermi-Fermi Mixtures of Open-Shell ${ }^{6} \mathrm{Li}$ and Closed-Shell ${ }^{173}$ Yb Atoms, Phys. Rev. X 10, 031037 (2020).

[39] R. Wynar, R. S. Freeland, D. J. Han, C. Ryu, and D. J. Heinzen, Molecules in a Bose-Einstein Condensate, Science 287, 1016 (2000).

[40] T. Rom, T. Best, O. Mandel, A. Widera, M. Greiner, T. W. Hänsch, and I. Bloch, State Selective Production of Molecules in Optical Lattices, Phys. Rev. Lett. 93, 073002 (2004).

[41] L. R. Liu, J. D. Hood, Y. Yu, J. T. Zhang, K. Wang, Y.-W. Lin, T. Rosenband, and K.-K. Ni, Molecular Assembly of Ground-State Cooled Single Atoms, Phys. Rev. X 9, 021039 (2019).

[42] D. J. Wineland, M. Barrett, J. Britton, J. Chiaverini, B. DeMarco, W. M. Itano, B. Jelenković, C. Langer, D. Leibfried, V. Meyer, T. Rosenband, and T. Schätz, Quantum Information Processing with Trapped Ions, Phil. Trans. R. Soc. A 361, 1349 (2003).

[43] M. Korek, S. Bleik, and A. R. Allouche, Theoretical Calculation of the Low Laying Electronic States of the Molecule NaCs with Spin-Orbit Effect, J. Chem. Phys. 126, 124313 (2007).

[44] A. Grochola, P. Kowalczyk, J. Szczepkowski, W. Jastrzebski, A. Wakim, P. Zabawa, and N. P. Bigelow, Spin-Forbidden $c^{3} \Sigma^{+}(\Omega=1) \leftarrow X^{1} \Sigma^{+}$Transition in NaCs: Investigation of the $\Omega=1$ State in Hot and Cold Environments, Phys. Rev. A 84, 012507 (2011).

[45] J. Zaharova, M. Tamanis, R. Ferber, A. N. Drozdova, E. A. Pazyuk, and A. V. Stolyarov, Solution of the Fully-MixedState Problem: Direct Deperturbation Analysis of the $A^{1} \Sigma^{+}-b^{3} \Pi$ Complex in a NaCs Dimer, Phys. Rev. A 79, 012508 (2009).
[46] A. Grochola, P. Kowalczyk, and W. Jastrzebski, Investigation of the $\mathrm{B}^{1} \Pi$ State in NaCs by Polarisation Labelling Spectroscopy, Chem. Phys. Lett. 497, 22 (2010).

[47] P. J. Zabawa, Production of Ultracold, Absolute Vibrational Ground State NaCs Molecules, Ph.D. Thesis, University of Rochester, 2012.

[48] L. R. Liu, J. T. Zhang, Y. Yu, N. R. Hutzler, Y. Liu, T. Rosenband, and K.-K. Ni, Ultracold Molecular Assembly, arXiv:1701.03121.

[49] F. H. Mies, E. Tiesinga, and P. S. Julienne, Manipulation of Feshbach Resonances in Ultracold Atomic Collisions Using Time-Dependent Magnetic Fields, Phys. Rev. A 61, 022721 (2000).

[50] J. D. Hood, Y. Yu, Y.-W. Lin, J. T. Zhang, K. Wang, L. R. Liu, B. Gao, and K.-K. Ni, Multichannel Interactions of Two Atoms in an Optical Tweezer, Phys. Rev. Research 2, 023108 (2020).

[51] L. R. Liu, J. D. Hood, Y. Yu, J. T. Zhang, N. R. Hutzler, T. Rosenband, and K.-K. Ni, Building One Molecule from a Reservoir of Two Atoms, Science 360, 900 (2018).

[52] K. Wang, X. He, R. Guo, P. Xu, C. Sheng, J. Zhuang, Z. Xiong, M. Liu, J. Wang, and M. Zhan, Preparation of a Heteronuclear Two-Atom System in the Three-Dimensional Ground State in an Optical Tweezer, Phys. Rev. A 100, 063429 (2019).

[53] This interaction shift is larger than the differential axial trapping frequency between $\mathrm{Na}$ and $\mathrm{Cs}$ atoms, which decouples the relative and center-of-mass motional state and improves the robustness of our preparation of the relative motional ground state.

[54] Y. Yu, Coherent Creation of Single Molecules from Single Atoms, Ph.D. Thesis, Harvard University, 2021.

[55] M. Endres, H. Bernien, A. Keesling, H. Levine, E. R. Anschuetz, A. Krajenbrink, C. Senko, V. Vuletic, M. Greiner, and M.D. Lukin, Atom-by-Atom Assembly of Defect-Free One-Dimensional Cold Atom Arrays, Science 354, 1024 (2016).

[56] D. Barredo, V. Lienhard, S. de Léséleuc, T. Lahaye, and A. Browaeys, Synthetic Three-Dimensional Atomic Structures Assembled Atom by Atom, Nature (London) 561, 79 (2018).

[57] D. O. de Mello, D. Schäffner, J. Werkmann, T. Preuschoff, L. Kohfahl, M. Schlosser, and G. Birkl, Defect-Free Assembly of 2D Clusters of More Than 100 Single-Atom Quantum Systems, Phys. Rev. Lett. 122, 203601 (2019).

[58] J. M. Hutson, Coupled Channel Methods for Solving the Bound-State Schrödinger Equation, Comput. Phys. Commun. 84, 1 (1994).

[59] J. M. Hutson and C. R. Le Sueur, MOLSCAT: A Program for Non-Reactive Quantum Scattering Calculations on Atomic and Molecular Collisions, Comput. Phys. Commun. 241, 9 (2019).

[60] J. M. Hutson and C. R. Le Sueur, MOLSCAT, BOUND, and FIELD, version 2020.0, https://github.com/molscat/molscat.

[61] J. M. Hutson and C. R. Le Sueur, BOUND and FIELD: Programs for Calculating Bound States of Interacting Pairs of Atoms and Molecules, Comput. Phys. Commun. 241, 1 (2019).

[62] O. Docenko, M. Tamanis, J. Zaharova, R. Ferber, A. Pashov, H. Knöckel, and E. Tiemann, The Coupling of the $x^{1} \sigma^{+}$and 
$x^{3} \sigma^{+}$States of the Atom Pair $\mathrm{Na}+\mathrm{Cs}$ and Modelling Cold Collisions, J. Phys. B 39, S929 (2006).

[63] M. Gröbner, P. Weinmann, E. Kirilov, H.-C. Nägerl, P. S. Julienne, C. R. Le Sueur, and J. M. Hutson, Observation of Interspecies Feshbach Resonances in an Ultracold ${ }^{39} \mathrm{~K}-{ }^{133} \mathrm{Cs}$ Mixture and Refinement of Interaction Potentials, Phys. Rev. A 95, 022715 (2017).

[64] J. M. Hutson, E. Tiesinga, and P. S. Julienne, Avoided Crossings between Bound States of Ultracold Cesium Dimers, Phys. Rev. A 78, 052703 (2008).

[65] A. E. Thornley and J. M. Hutson, Bound-State Wave Functions from Coupled Channel Calculations Using Log-Derivative Propagators: Application to Spectroscopic Intensities in Ar-HF, J. Chem. Phys. 101, 5578 (1994).
[66] E. Fattal, R. Baer, and R. Kosloff, Phase Space Approach for Optimizing Grid Representations: The Mapped Fourier Method, Phys. Rev. E 53, 1217 (1996).

[67] M. Tomza, W. Skomorowski, M. Musiał, R. González-Férez, C. P. Koch, and R. Moszynski, Interatomic Potentials, Electric Properties and Spectroscopy of the Ground and Excited States of the $\mathrm{Rb}_{2}$ Molecule: Ab Initio Calculations and Effect of a Non-Resonant Field, Mol. Phys. 111, 1781 (2013).

[68] I. Klincare, J. Zaharova, M. Tamanis, R. Ferber, A. Zaitsevskii, E. A. Pazyuk, and A. V. Stolyarov, Radiative Lifetimes of the (1-3) ${ }^{1} \Pi$ States in NaCs: Experiment and Theory, Phys. Rev. A 76, 032511 (2007).

[69] M. Aymar and O. Dulieu, Calculations of Transition and Permanent Dipole Moments of Heteronuclear Alkali Dimers NaK, NaRb and NaCs, Mol. Phys. 105, 1733 (2007). 